\title{
Reducing uncertainty in practical radiation thermometer calibration based on proposed changes to the mise en pratique of the definition of the kelvin
}

\author{
David Lowe $^{1}$, Mick Broughton ${ }^{2}$ and Jon R Willmott ${ }^{2}$ \\ ${ }^{1}$ National Physical Laboratory, Hampton Road, Teddington, Middlesex TW11 0LW, UK \\ ${ }^{2}$ Land Instruments International, Stubely Lane, Dronfield, Derbyshire S18 1DJ, UK
}

\begin{abstract}
The effect of changing calibration traceability for radiation thermometry from the current International Temperature Scale of 1990 (ITS-90) to a proposed SI based scale is investigated in the context of the dissemination of a radiation thermometer based high temperature scale from national measurement institute to calibration laboratory. It is shown that at temperatures up to $2500{ }^{\circ} \mathrm{C}$ the uncertainty in temperature scale realisation can be halved without changing the calibration laboratory methodology. Significant benefits are possible now, based on present ITS-90, in advance of any proposed changes. The requirements for a suitable radiation thermometer to achieve this are considered.

L'effet de changement de traçabilité de l'étalonnage de ITS-90 à un projet de barème basé SI est étudiée dans le cadre de la diffusion d'une échelle à haute température de l'institut national de mesure des laboratoires d'étalonnage. Il est montré que, à des températures allant jusqu'à $2500{ }^{\circ} \mathrm{C}$ l'incertitude dans la réalisation de l'échelle de température peut être réduite de moitié sans changer la méthode de laboratoire d'étalonnage. Les exigences pour un pyromètre adapté pour atteindre cet objectif sont considérés.
\end{abstract}

\section{Introduction}

The standard route for traceable non-contact temperature measurement in the UK is to have a radiation thermometer (transfer standard) calibrated by comparison to a primary standard radiation thermometer at the UK's national measurement institute (NMI), the National Physical Laboratory (NPL). Calibration is in terms of the International Temperature Scale of 1990, ITS-90. This calibrated radiation thermometer can then in turn be used in turn as a standard to calibrate other instruments, with a traceable chain of calibration from national standards to end user. The process of calibrating the primary standard involves determining the spectral characterisation of a non-linearised radiation thermometer (i.e. the basic output is linear with the source radiance, not linear with temperature). The signal at an ITS-90 fixed point (the freezing temperature of silver, gold or copper) is used as a reference. The linearity, ambient sensitivity and optical imaging quality of the primary standard thermometer have to be assessed as well to allow a full uncertainty budget to be determined. The primary standard can then be used to assign a temperature to a variable high temperature blackbody source, which can then be used as a source to calibrate a transfer standard. In this way the ITS-90 temperature scale is transferred from the primary instrument to the secondary or transfer standard. In this study we will consider the calibration of a transfer standard to be used in the calibration laboratory at Land Instruments International (LII). The calibrated transfer standard is used at LII to assign a temperature to their variable temperature blackbody source. This process is then repeated to calibrate a series of working standards, each of which is a production type and is used to calibrate the corresponding devices for industrial customers. The geometry is carefully arranged from NMI onward such that all LII instruments are viewing an equivalent field of view, the nominally $15 \mathrm{~mm}$ diameter central region on the backwall of the variable temperature blackbody source. This greatly simplifies the calibration process for LII but does place some restrictions on what can be changed to reduce uncertainties.

In the next few years the kelvin is to be redefined in terms of the Boltzmann constant instead of the triple point of water [1]. It is expected that at the same time additional routes will be introduced to the mise en pratique of the definition of the kelvin (MeP-K) that qualify as being traceable to national standards, and so will be acceptable alternatives to ITS-90 [2]. This opens the possibility that changes could be made to the calibration chain that would result in reduced uncertainties for the end user without disturbing the basic process of measurement at the calibration laboratory. One expected change is to be the inclusion of temperatures and uncertainties of high temperature fixed points 
(HTFPs). These could be used to directly calibrate a transfer standard, bypassing the primary standard and variable temperature blackbody route. To assess the potential benefits to a calibration laboratory a simple and relatively low cost radiation thermometer was calibrated using high temperature fixed points with an uncertainty budget based both on the expected outcome of current Tassignment measurements [3], and also on current ITS-90 capability. This thermometer was then used at LII to determine the uncertainties that might be achievable in assigning a temperature to their blackbody source in comparison to the current route.

\section{Calibration}

The LII transfer standard (the NQO) is calibrated at NPL in terms of ITS-90 and is issued with a UKAS (United Kingdom Accreditation Service) certificate ensuring traceability to national standards. The NQO is a simple system of a lens in a tube focusing on a silicon photodiode with a broadband spectral response 0.5 to $1.1 \mu \mathrm{m}$ The trial pyrometer (denoted P715 [4]) was calibrated in terms of the expected mise en pratique of the definition of the kelvin. This pyrometer has a combination of coloured glass filters to give a bandpass centred at $715 \mathrm{~nm}$ and a fwhm of $76 \mathrm{~nm}$. It uses a Hamamatsu S1337-21 silicon photodiode and a Vinculum E690 transimpendence amplifier with variable $10^{6}, 10^{7}$ or $10^{8}$ volts/amp gain.

Figures 1(a) and 1(b) summarise the two schemes used; the current calibration process and possible schemes based on fitting to a series of HTFPs

\subsection{Current calibration to ITS-90}
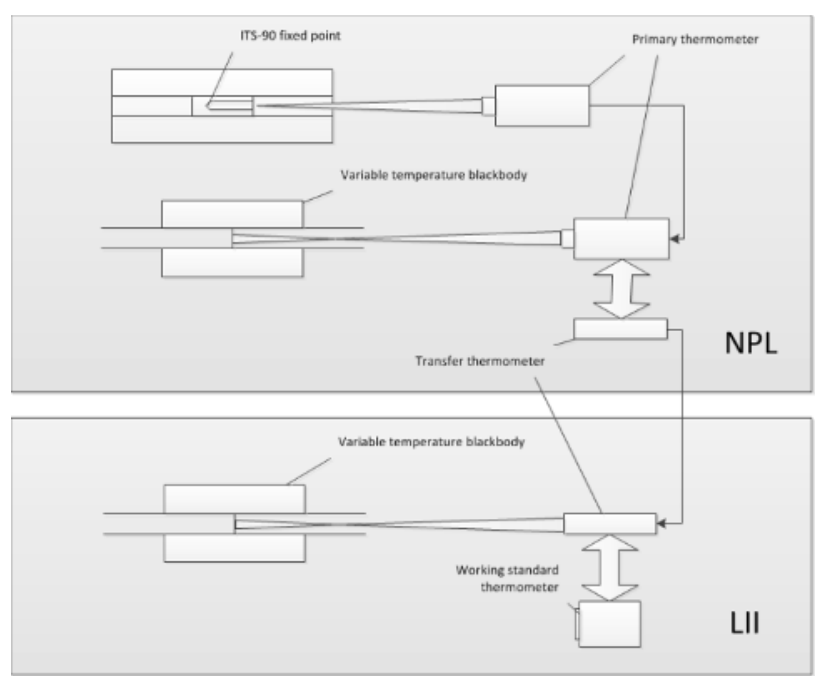

Figure 1(a) The current scheme: a standard thermometer is calibrated in terms of ITS-90. A transfer standard is calibrated by comparison using a variable temperature source. Working standards are then calibrated by comparison to the transfer standard.

The current approach, illustrated in Figure 1(a), uses a very stable and linear radiation thermometer with a welldefined narrow bandpass spectral response (an IKE LP3) calibrated in terms of ITS-90 and a copper fixed point is used as reference. Calibration of a transfer standard is by comparison to the LP3 using a Thermogauge $25 \mathrm{~mm}$ diameter blackbody. To calibrate the NQO the temperature and uniformity of the blackbody backwall over the central $15 \mathrm{~mm}$ is determined using the LP3. The NQO views this portion of the source. It is calibrated from $1000{ }^{\circ} \mathrm{C}$ to $2500{ }^{\circ} \mathrm{C}$. The NQO is well behaved and gives lower uncertainties than most other pyrometers calibrated at NPL. It also has a long history and is known to be extremely stable. It has a nominal target size of $1 \mathrm{~mm}$ diameter but during calibration, and in use, it is moved back such that it views an area approximately $15 \mathrm{~mm}$ diameter.

The uncertainties of the calibration consist of:

- the uncertainty of the source radiance temperature

- combined reproducibility of the calibrated LP3 and Thermogage radiance source

- scale realisation with the LP3

○ uniformity of the source

- the uncertainty in the NQO output

- reproducibility of the NQO

- short term repeatability of the calibration

- uncertainty in the evaluation of the size-of-source effect)

- the effect of differences between the LP3 and NQO

$\circ$ emissivity of the source and the difference in wavelength between the NQO and the LP3

- effect of alignment and mismatch of field of view.

The size-of-source effect measurements are reported in the certificate but corrections are not needed as the geometry used is kept constant at all stages.

\subsection{Calibration by high temperature fixed points}

A pyrometer can already be calibrated using htfps, with ITS-90 temperatures assigned to the fixed points by a calibrated primary pyrometer, but the full benefit is expected to come when low uncertainty $\mathrm{T}$ values are assigned. The P715 thermometer was designed to be calibrated using these high temperature fixed points: it has small target size and good size-of-source effect. The intention is that such a device would be simple enough that it could be supplied as a calibrated standard directly from an NMI with a level of uncertainty comparable to calibration measurement capability $(\mathrm{CMC}$, for NPL this is $0.05 \%$ of temperature above $\left.1000{ }^{\circ} \mathrm{C}, k=2\right)$. The P715 thermometer was calibrated at fixed points of cobaltcarbon (nominally $1324^{\circ} \mathrm{C}$ ), platinum-carbon $\left(1738^{\circ} \mathrm{C}\right)$ and rhenium-carbon $\left(2474{ }^{\circ} \mathrm{C}\right)$. Two uncertainty budgets were prepared: one using target uncertainties for current research [3] of $0.2{ }^{\circ} \mathrm{C}$ at the cobalt-carbon point, $0.3{ }^{\circ} \mathrm{C}$ at platinum-carbon and $0.4{ }^{\circ} \mathrm{C}$ at rhenium-carbon, and one using the uncertainty when assigning temperatures using current ITS-90 capability. The uncertainty budget 
components in assigning a temperature and signal at each fixed point are based on the Comité consultatif de thermométrie (CCT) recommendations [5] and the propagation of the uncertainties between fixed points follows [6]. A full description of this radiation thermometer with values is given in [4]. This approach is illustrated in Figure 1(b)

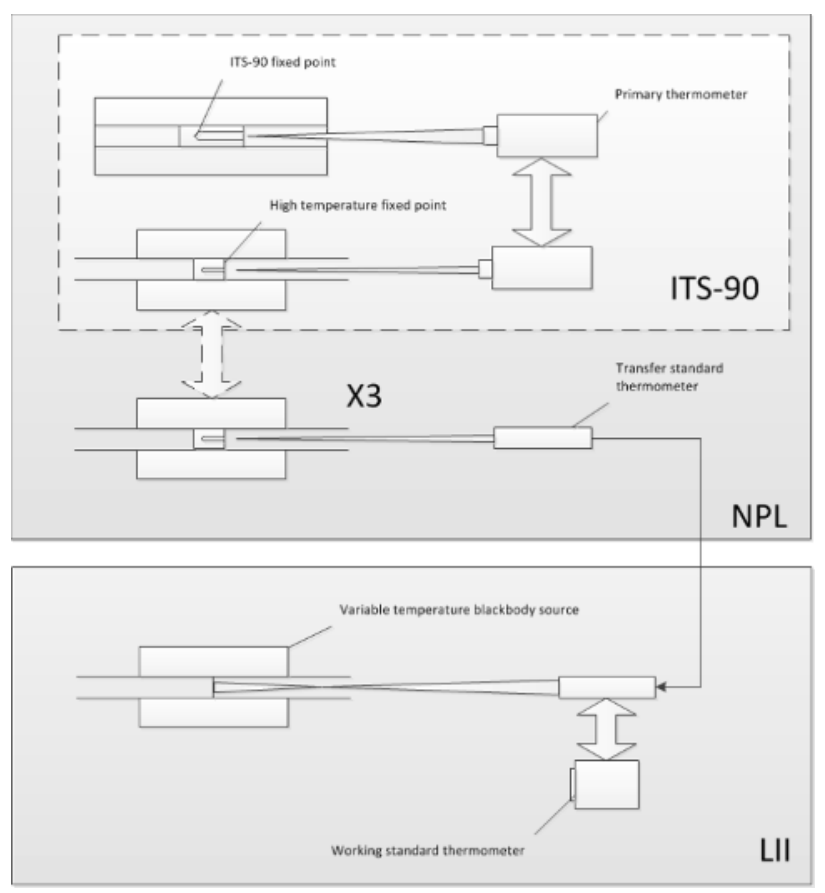

Figure 1(b) High temperature fixed points have ITS-90 temperatures assigned. In future they will have assigned temperatures with agreed uncertainties. A suitable pyrometer is calibrated and then used to calibrate working standards by comparison.

\subsection{Size-of-source response}

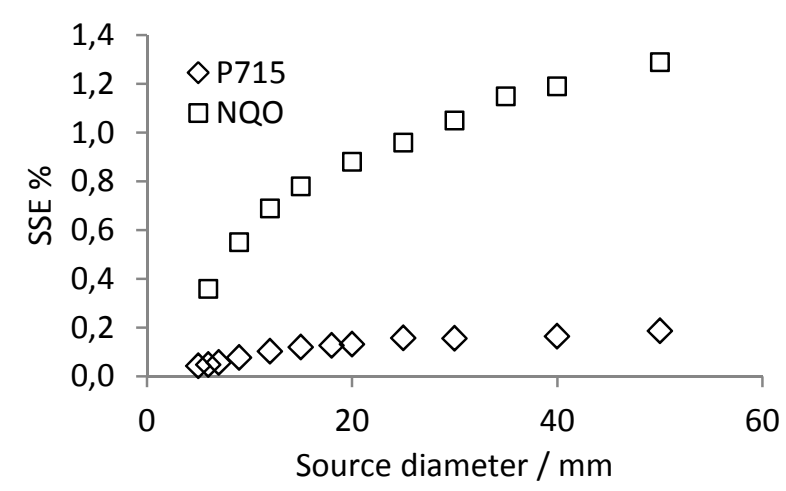

Figure 2. Size-of-source effect: the additional signal detected from a uniform source above that measured at a source diameter of $3 \mathrm{~mm}$.

The use of HTFPs ideally requires a field of view (fov) less than half the fixed point aperture, in this case a fov of less than $1.5 \mathrm{~mm}$ with the $3 \mathrm{~mm}$ diameter blackbody sources, and to have good size of source effect (SSE). That is, that the thermometer is not significantly influenced by the radiance of the furnace used to melt the fixed-point. The NQO is always used under the same viewing geometry and so the $S S E$ correction can be ignored. Figure 2 compares the results for P715 and the NQO. For the P715 thermometer, since the fov is different between calibration and use, the difference between $3 \mathrm{~mm}$ (the HTFPs aperture diameter) and $15 \mathrm{~mm}$ (the in-use geometry) is taken as the uncertainty.

\subsection{Linearity}

The linearity of the P715 was assessed using a radiance doubling technique. It was found to be linear to within $0.05 \%$ up to an output equivalent to $2500{ }^{\circ} \mathrm{C}$. The ratios between gain ranges $10^{6}, 10^{7}$ and $10^{8} \mathrm{~V} / \mathrm{A}$ were assessed by measuring the HTFPs at different gain settings.

\subsection{Stability}

The NQO has a long history over several decades, and has proved to be very stable. It is calibrated at three year intervals. The P715 is a recently constructed developmental model and stability is less well known. For the P715 fixed points measurements have been made 18 months apart. Although these measurements include the scale realisation uncertainty of the P715 thermometer itself, they allow an estimate of an upper limit on long term stability.

\subsection{Wavelength bandwidth}

The MeP-K approach requires fitting a function to allow interpolation between the fixed points. The SakumaHittori equation was used [7] which ideally requires a narrow bandpass spectral response. By comparing a fit to three fixed points with the integrated response of the filter and Planck function a correction and/or uncertainty can be evaluated.

\section{Uncertainty Comparison}

Measurements were performed at LII with their Thermogauge $25 \mathrm{~mm}$ dual blackbody calibration source. The NQO is the top level standard used for UKAS accredited calibration and so has a well established uncertainty budget including evaluated components for source uniformity and stability. The components in assigning a temperature to the furnace consist of:

- Ws: The calibration of the NQO, from the calibration certificate

- Ds: The drift of the NQO between calibrations. To make the comparison on an equal basis this was halved from the normal value to be equivalent to the 18 months P715 data. Since the NQO stability is one of the smaller components doing this does not distort the overall budget.

- Ps: Alignment - an allowance based on the uniformity of the source and how well the NQO is aligned to cover the possibility that the NQO may not be viewing the same area on the back wall as the unit under test (UUT) being calibrated 
- As: Ambient temperature correction - the sensitivity to the temperature of the laboratory

- Rs: Repeatability - the intrinsic repeatability of the NQO. Assessed by assuming the source is stable over a short period and measuring the standard deviation of the output

- Reu: Digital multi-meter rounding - the resolution of the voltmeter

- Ms: Digital multi-meter specification - the uncertainty of the voltmeter

- Ls: Leads effect - there is a small change in the signal due to the leads from the NQO to the digital multi-meter

- Ts: Table interpolation. The NQO is calibrated at intervals. Values in between are determined by linear interpolation. In reality the Planck function should be used.

- Ss: Source stability - the change in the source temperature over the course of a calibration point.

This uncertainty budget was used as the basis for the measurements with the P715 thermometer. The changed components were:

- As: The P715 is temperature stabilised. The effect of $1^{\circ} \mathrm{C}$ change on output was used.

- Ts: The P715 thermometer is calibrated to give an equation relating output to temperature, so there is no interpolation error.

Otherwise the LII standard uncertainty budget was used. This allows us to see what would happen if the current LII standard were to be replaced with an instrument calibrated on the basis of a future MeP-K.

\section{Results}

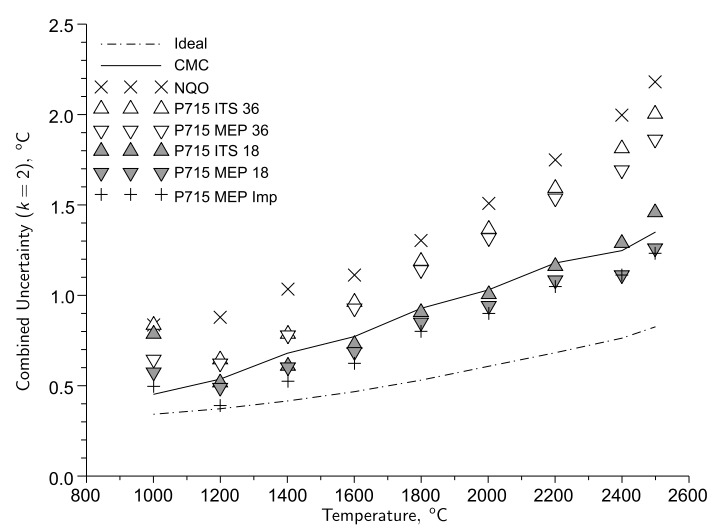

Figure 3 The combined uncertainty $(k=2)$ in assigning a temperature to the LII calibration furnace given various different scenarios.

Figure 3 shows the uncertainties achieved in assigning temperatures to the LII Thermogauge blackbody source for a number of different cases. This is the uncertainty in the source that will then be a contributor to calibration of the secondary standards. The uncertainty components relating to the source itself, the alignment and the use of ancillary equipment have been left the same for all cases. Only the contributions due directly to the thermometer have been changed.

Two benchmark curves are plotted. One ("Ideal") is for an uncertainty budget based on a thermometer with zero uncertainty. That is, it is the uncertainty associated with the source rather than with the assignment of a temperature to that source that is dominant. It is the floor to which the uncertainties would tend as the calibration and in-use uncertainties of the thermometer became negligible. The other ("CMC") is based on NPL's accredited CMC capability. It is the calibration uncertainty that NPL can demonstrate to an independent assessor is routinely available given an adequate thermometer. That is, it is the potential minimum NPL contribution to the uncertainty budget of a customer calibration. Therefore the curve for CMC shows the uncertainty in temperature of the blackbody source using the best UKAS accredited uncertainty NPL would currently be able to supply with the P715 instrument.

Figure 3 then shows a number of cases. "NQO" is the current situation based on the NQO thermometer with a calibration interval of 3 years. This is what is achieved now, and is the basis of all LII high temperature calibrations. The dominant uncertainty contributions are the calibration uncertainty (Ws) of the NQO and its sensitivity to environmental conditions (As). The drift (Ds), repeatability (Rs) and interpolation (Ts) are minimal in comparison.

There are then four cases for the P715; two showing differences in the way htfps could be specified and two showing different calibration intervals. "P715 ITS 36" shows how the P715 would behave as a direct replacement given the situation as it is now. Calibration is in terms of ITS-90, with the fixed points used for the calibration having their temperatures determined by the NPL calibrated standard pyrometer. A calibration interval of three years, the same frequency as the NQO, was assumed. Since there has been insufficient time to establish the stability of the P715 to any degree of certainty, drift is the dominant uncertainty component. The overall uncertainty is slightly less than for the NQO, but not significantly so. The same scenario, but with the htfps having assigned temperatures on the basis of a possible mise en pratique is then considered ("P715 MEP 36"). This assumes defined uncertainties of $0.2^{\circ} \mathrm{C}$ at cobalt-carbon, $0.3{ }^{\circ} \mathrm{C}$ at $\mathrm{Pt}-\mathrm{C}$ and $0.4{ }^{\circ} \mathrm{C}$ at Re-C $(k=2)$ Again there is a small but not particularly significant reduction. Again the drift is the major component.

To see what the case would be if the drift were less than the measured limit the P715 results are shown recalculated for an 18 month recalibration interval (equivalently to a halving of the drift inferred from changes in fixed point measurements). ("P715 ITS 18" and "P715 MEP 18") There is now a significant reduction in the uncertainty for both ITS-90 and MeP-K calibration traceability schemes. Both show uncertainties at the level of NPL's best measurement capability.

Finally "P715 MEP IMP" shows what would be the case if this thermometer was found to be as stable as the 
NQO. The removal of any drift has minimal impact on the overall uncertainty budget. The case that the NQO had its calibration interval reduced was considered but since the drift is so small this has almost no effect.

\section{Discussion}

The current scheme has been chosen for its flexibility and simplicity. All the calibrations are of fixed geometry throughout the product range and turnaround of customer instruments is rapid. Any changes to reduce uncertainties should fit in with this scheme. The first thing to note is that a fairly simple instrument using standard components and calibrated using HTFPs gives uncertainties comparable to an extremely stable standard with decades of history that has some of the lowest uncertainties that NPL has delivered. The major issue of the new instrument is that of stability - so the next thing to note is that if this can be achieved then substantial improvements can be made in a calibration laboratory without the need to make any changes to the methodology. There comes a point where improvements to stability have no further improvement to the overall uncertainty of the source. The requirements for a drift at the uncertainty of calibration demonstrated here for P715 are for long term stability to be at or less than the uncertainty associated with the reference sources. That is, the stability can still be the major component in terms of the pyrometer, but the calibration interval should be such that the drift is not more than the "ideal" curve in Figure 3.

Whether the calibration is in terms of ITS-90 or in terms of the SI through an updated mise en pratique makes little difference with the methodology used here.

\section{Conclusions}

The use of a calibration scheme based on high temperature fixed points has the potential to halve the uncertainty of non-contact temperature scale realisation for a calibration laboratory. It is largely a matter of convenience which traceability route (ITS-90 or direct to the SI through a future mise en pratique) is used. As far as the NMI is concerned, a suitable instrument requires small target size, low SSE and accurate alignment (to the level of tenths of a millimetre) to be compatible with the fixed points. From the perspective of the calibration laboratory (at least in this study), standardisation of set up makes the optical performance less important, but stability needs to be good, to keep the recalibration schedule to a minimum. A ready guide to the stability needs is given by assessing the remaining uncertainty of the system assuming an ideal pyrometer with no uncertainty and no drift. If the drift is at or below this level it is not dominating the uncertainty budget. In contrast to the national measurement institute where alignment is critical, the calibration laboratory alignment tolerance is much less: the use of a kinematic mount can make it simple to set up once and then remove and replace the standard with sufficient accuracy.

To continue this work NPL is building pyrometers based on the P715 with refinements to improve stability (low expansion optical mounting, low thermal expansion field stop, UV protected wavelength defining filters and improved temperature stabilisation), and one of these will be assessed over the long term by LII.

\section{Acknowledgements}

The NPL author acknowledges funding from the National Measurement Office, an Executive Agency of the UK Department of Business, Innovation and Skill

\section{References}

[1] I. Mills, P. Mohr, T. Quinn, B. Taylor and E. Williams, Metrologia, 43, 227 (2006)

[2] D. Ripple, R. Davis, B. Fellmuth, J. Fischer, G. Machin, T. Quinn, P. Steur, O. Tamura and D. R. White, Int. J. Thermophys., 31, 1795 (2010)

[3] G. Machin, P. Bloembergen, J. Hartmann, M. Sadli and Y. Yamada, Int. J. Thermophys, 28, 1976 (2007)

[4] D. Lowe, Measurement Science and Technology, 24, 015901 (2012)

[5] CCT-WG5: Radiation thermometery, "CCT/03-03 Uncertainty budgets for realisation of scales by radiation thermometry," BIPM (2003)

[6] CCT-WG5: Radiation thermometery, “CCT/1012/rev1 Realisation and dissemination of thermodynamic temperature above the silver point," BIPM (2010) 